\title{
Dynamic portfolio optimization based on reference trajectories
}

\author{
Andrzej M.J. Skulimowski \\ Institute of Automatic Control, University of Mining 83 Metallurgy \\ Cracow, Poland
}

\begin{abstract}
We propose a multicriteria model for the dynamic multi-stage portfolio optimization. The information available at each decision step is applied to attain simultaneously the maximal rate of return, minimal risk, and the maximal investment flexibility by an appropriate selection of an optimal compromise decision. The input data concerning the portfolio structure and the state of economical environment at each moment of time is provided in form of the data matrices, transformed then to a state vector, only a part of it being known to the decision-maker. The evolution of the portfolio structure is described as a discrete-time control system, with the sell/buy transactions as controllable events. Missing coefficients are estimated using the Kalman filtering techniques. The investor's goals are modelled as reference points in the space of the criteria values for different planning horizons. To find the best-compromise allocation strategy we propose a decision-making method applying multiple reference points in dynamic multicriteria problems. This method can be implemented as an on-line interactive decision support system.
\end{abstract}

\section{Keywords}

Multicriteria optimization, portfolio theory, dynamic programming, discrete-time control systems.

\section{INTRODUCTION}

This paper addresses the problem of dynamic selection of the portfolio structure so that the criteria of maximal rate of return, minimal risk, maximal investment flexibility, and the demanded consumption curve were simultaneously taken into account while looking for an investment strategy. We apply consequently the multicriteria approach, so that the method proposed should be flexible enough to model the preferences of a possibly large class of investors, being thus a universal tool for a portfolio manager dealing with investors characterized by diversified risk attitudes. The desired consumption distribution over time serves at the same time as a trajectory objective and a reference trajectory in the multicriteria decision-making problem. As a special feature of the decision support model here presented, we allow to calculate the current value of portfolio and those of returns in several currencies simultaneously. Further, the user of the system is allowed to create his/her own artificial currencies fitting best his/her particular utilities.

Research supported in part by Swiss National Fund for Scientific Research, Grants No.12-30240.90 and 21-0377738.93/1. 
In the sequel we will admit no special limitation regarding the portfolio structure under consideration, which may contain stocks, corporate bonds, government bonds, treasury bills, precious metals, real estate, and liquidity, as well as options and futures on stocks, currencies, composite indices, bonds, and metals. Nevertheless, we will concentrate our attention on pure security portfolios, especially stocks and options on stocks and currencies. Each of the above will be referred to as a portfolio category, which in the sequel may be subdivided into the country of issue, place of trade, the currency of trade, and the physical place of storing or the bank account where it is deposited.

The portfolio structure for each moment from the time interval considered is described in form of the state vector in a discrete-time control system. The data concerning the economical environment used in the optimization process are stored in the data matrices, being known only partly to the decision-maker. Missing coefficients are estimated using the Kalman filtering techniques. The investor's goals are modelled as reference points in the space of the criteria values for different planning horizons. To find the best compromise allocation strategy we propose a decision-making method applying multiple reference points in dynamic multicriteria problems. The method can be implemented as an on-line interactive decision support system.

Summarizing, the main task of the decision support system proposed is to provide the decision-maker with a possibly best compromise investment strategy, taking into account its investment goals, risk aversion (or attitude), an attitude to make flexible investment decisions, and the possibility of adaptively modifying the selected investment strategy in a response to the changes of the environment. Such strategy will be derived based on economic data such as past and current prices, price expectations, turnover etc., provided by the decision-maker from external knowledge bases and evaluated within the system.

\section{PRINCIPLES OF PORTFOLIO MODELLING}

Following the above remarks, the physical structure of portfolio at a moment $t$ will be described by the vector $x(t) \in \mathbb{R}^{n}$. The unit prices for each portfolio category are expressed in $m$ different currency units and are contained in the real matrix $P(t) \in M_{n(t), m(t)}$, $\mathrm{i}$-th row of $P(t)$ containing the prices in the $i$-th currency for $i=1, . . m(t)$.

By the portfolio transformations we will mean the sell/buy transactions, the generation of options, the expiration and exercising of options and futures, the conversion of convertible bonds and warrants, and some other processes. The portfolio transformations are described by the equation

$x(t+1)=A(t) x(t)+B(t) u(t)+\xi(t)$

with the matrix $A(t)$ determined by the transformations which cannot be influenced by the decision-maker, while the control variables $u(t)$ can be fully determined, perhaps subject to a set of constraints. The matrix $B(t)$ models the control laws, and $\xi(t)$ is the stochastic disturbance which can be interpreted as unexpected gains or losses. The evolution of prices may be described by the following rule

$P(t+1)=\psi(P(t), \varepsilon(t), \zeta(t))$

where $\psi$ is the price formation mechanism (usually known only partly), $\varepsilon(t)$ is the state vector of economic environment, which may also be at least in part unknown, and $\zeta(t)$ is 
the random disturbance factor. Thus the increase $\Delta V(t, t+1)$ of the portfolio value $V(t)$ on the time interval $[t, t+1)$, is given by the formula

$\Delta V(t, t+1):=V(t+1)-V(t)=$

$$
=\left[P^{T}(t+1) A(t)-P^{T}(t)\right] x(t)+R(t, t+1)+P^{T}(t) b(t)-C(t-1, t)-P^{T}(t) s(t),
$$

where $b(t)$ is the vector of buys, $s(t)$ - the vector of sales, and $R(t-1, t)$ and $C(t-1, t)$ are the direct net revenue (such as dividends or interests), and consumption vectors for the period $(t-1, t]$, respectively. Throughout the paper the symbol $A^{T}$ will denote the transposition of the matrix $A$.

Each of $\mathrm{m}$ rows of $P(t), p_{j}(t)$, as well as each coordinate $r_{j}(t)$ of $R(t)$ corresponds to a monetary unit the current value may be expressed in. As the monetary units one can use real currencies, each one of portfolio categories, artificial currencies such as ECU, etc. In the most common case of one privileged monetary unit, $P(t)$ consists of a single price vector $p(t)$. The coordinates of the vector $x(t)$ may be interpreted either as physical quantities of each portfolio category, e.g. number of stocks of specific companies, weight of gold, amount of cash etc., in this paper called items, or as estimated values of real estate.

For the sake of simplicity, in the sequel we will assume that the decision-maker is able to choose one of the currencies, or define own value function, so that one can employ the scalar measure $\mathrm{v}$ for the portfolio value. Hence (3) can be rewritten as $\Delta v(t, t+1):=v(t+1)-v(t)=$

$$
=\left[p^{T}(t+1) A(t)-p^{T}(t)\right] x(t)+R(t, t+1)+p^{T}(t) b(t)-C(t-1, t)-p^{T}(t) s(t),
$$

where $v(t):=\phi(V(t)), \quad r(t-1, t):=\phi(R(t-1, t)), c(t-1, t):=\phi(C(t-1, t))$ for certain aggregating currency function $\phi: \mathbb{R}^{n} \rightarrow I R$. As an example of $\phi$ may serve the projection on one of the coordinates corresponding to the selection of a single currency, or a linear combination of the coordinates which imposes the book-keeping in an artificial currency such as ECU.

\subsection{Modelling the portfolio transformations}

The revenue vector $R$ may be represented as the sum

$R(t-1, t)=\sum_{i=1}^{n} r_{k}(t-1, t)$,

where $r_{k}, \quad k=1, . . n$, is the revenue vector coming from the $k$-th portfolio category. On the other hand, if the portfolio structure changes according to

$x(t)=A(t-1, t) x(t-1)+\sum_{k=1}^{n}\left[x_{k j+}(t-1, t)-x_{k j-}(t-1, t)\right]$,

where $A(t-1, t)$ is the growth rate matrix, then the total revenue on the interval $[t-1, t)$ equals to

$\dot{R}(t-1, t)=P^{T}(t) x(t)-P^{T}(t-1) A(t-1, t) x(t-1)$.

The values of

$s_{k j}(t-1, t):=r_{k j}(t-1, t) /\left(M_{k j}(t-1) x_{k}(t-1)\right)$

express the net rate of return of $k$-th portfolio category expressed in the $j$-th monetary unit, for $k=1, . . n, j=1, . . m$. At each moment of time $t$ the structure of the portfolio vector $x(t)$ may be transformed according to the constraints

$\sum_{k=1}^{n} x_{k j+}(t-1, t) p_{k j+}(t-1, t) \leq$ 


$$
\leq \sum_{k=1}^{n} x_{k j-}(t-1, t) p_{k j-}(t-1, t)+r_{j}(t-1, t)+d_{j}(t-1, t), \quad \text { for } j=1, . . m,
$$

where $P_{+}(t-1, t)$ and $P_{-}(t-1, t)$ are the matrices of minimal buy, and maximal sale prices for each portfolio category over the period $[t-1, t]$, respectively, $d_{j}(t-1, t)$ is the debt in the $j$-th monetary unit and $x_{k j+}(t-1, t)$ and $x_{k j-}(t-1, t)$ are the amounts of each items bought, or resp. sold over the period $[t-1, t]$ and fulfilling the balance equations

$x_{k}(t)=x_{k}(t-1)+\sum_{j=1}^{m}\left(x_{j k+}(t-1, t)-x_{j k-}(t-1, t)\right), \quad$ for $k=1, . . n$.

A simple exchange transaction consisting in selling the quantity $x_{k j-}(t-1, t)$ from the $k$-th portfolio category at price $p_{k j-}(t-1, t)$ and buying an equivalent amount of the $i$-th item, $x_{i j+}(t-1, t)$, at the price $p_{i j+}(t-1, t)$ expressed in the same $j$-th monetary unit may be written as

$x_{i j+}(t-1, t)=p_{k j-}(t-1, t) / p_{k j+}(t-1, t) x_{k j-}(t-1, t)$.

The mixed exchange transaction involving the payment in various monetary units and investing a part of the revenue may be expressed as

$x_{k+}(t-1, t)=\sum_{j=1}^{n}\left[\sum_{i=1}^{m} p_{i j-}(t-1, t) x_{i k j-}(t-1, t) / p_{k j+}(t-1, t)+r_{j}(t-1, t) / p_{k j+}(t-1, t)\right],(8)$

where $x_{i k j+}(t-1, t)$ denotes the quantity of $i$-th item sold for the j-th monetary unit and used for buying $k$-th item.

Arbitrary change of the structure vector between the moments $t-1$ and $t$ according to the constraints (8) will be called investment. It is easy to see that each investment can be represented as the superposi- tion of mixed exchange transactions.

In the sequel we will assume that the structure of the portfolio vector at moment $t$ may be influenced by the values of $x(t-1)$, which may be expressed e.g. as the growth rate constraints

$x_{i}(t)-x_{i}(t-1) \leq \sum_{j \in J} \lambda_{j}(t) x_{j}(t-1)$ for certain $J \subset\{1, \ldots n\}$.

The coefficients $\lambda_{j}(t)$ represent the relative amounts of $j$-th items in the portfolio which can be used for buying the $i$-th items at the moment $t$.

\subsection{Description of portfolio evolution as a discrete-time control system}

An $r$-th complex transaction protocol may be available as the transition form $\Gamma_{r}(t, t+1)$, i.e the matrix

$\left[q_{i j}^{r}\right]_{1 \leq i \leq N(t), 1 \leq j \leq N(t+1)}$

The value of $q_{i j}^{r}$ denotes the (physical) amount of the $i$-th portfolio category changed for the $j$-th category on the interval $[t, t+1)$ within the $r$-th transaction, and $N(t)$ denotes the total amount of portfolio classes considered. Usually, by selling the various portfolio catego- ries an amount $q_{\beta \nu}(t, t+1)$ of the $\beta$-th category will be exchanged for liquidity $b_{\nu}(t+\tau)$, and an amount of $p_{\beta \nu}(t) q_{\beta \nu}(t, t+1)$ will be located on the $\nu$-th account after the time lapse $\tau$. Analogously, a buy consists in replacing the liquidity $b_{\nu}(t+\tau)$ by a specified amount of the $\mu$-th portfolio category. For the sake of simplicity we will consider immediate buys only. 
The aggregated transaction matrix is the matrix $\Theta(t, t+1)=\left[q_{i j}(t, t+1)\right]_{1 \leq i \leq N(t), 1 \leq j \leq N(t+1)}$

derived from the matrices $\Gamma_{r}(t, t+1), \quad r=1, \ldots \rho$. It contains the absolute values of changes of each portfolio category on $[\mathrm{t}, \mathrm{t}+1)$.

The relative values $r_{i j}(t, t+1):=q_{i j}(t, t+1) / x_{i}(t)$ will be stored in the matrix $A_{2}(t)$ of the same dimensions that $\Theta$. It is easy to see that then we get the equation

$x(t+1)=A_{1}(t) x(t)+A_{2}(t) \Theta(t, t+1) e+\xi(t)$

where $e=(1, . .1) \in \mathbb{R}^{N(t)}$ and $\xi(t)$ is the random perturbance representing the unexpected losses or gains, which constitutes the simplest form of the state-space portfolio model. Observe that the matrix $A_{1}$ need not be quadratic as the dimensions $N(t+1)$ and $N(t)$ of $x(t+1)$ and $x(t)$, respectively, may be different in case where new portfolio category is appended to the portfolio vector between $t$ and $t+1$, or if another one is not to be considered any more. The next step consists in distinguishing a subset of controlled actions and transforming the system to the form

$x(t+1)=A(t) x(t)+B(t) u(t)+w(t)+\xi(t)$,

where $u(t)$ are controls and $B(t)$ is the control matrix describing the control laws. As non-controlled events $w(t)$ should be regarded those transactions which are imposed to the decision-maker. as e.g. the obligatory future realization after assignment, while all those being freely chosen are controls.

\section{FORMULATION OF THE MULTICRITERIA OPTIMIZATION PROBLEM}

Below we will show an application of reference points in the criteria space to select a satisfactory solution to the portfolio management problem at each step of the optimization process.

The problem of choice of an optimal portfolio structure at a moment $t$ will be modelled as the multicriteria optimization problem

$\left(F_{t}: U \rightarrow \mathbb{R}^{N}\right) \rightarrow \min$

where $U$ is the decision space, $I^{N}$ the criteria space, and $F_{t}(u)=\left(F_{1}(t, u), F_{2}(t, u), \ldots\right.$, $\left.F_{N}(t, u)\right)$ is a vector objective function for the decision step $[\mathrm{t}, \mathrm{t}+1)$. Let us recall that a solution $u_{0}$ to the multicriteria optimization problem (13) is called Pareto-optimal, or nondominated, iff the admission of no other control results in the values of $F_{1}, \ldots F_{N}$ being all better than those achieved for $u_{0}$, i.e. for the simultaneous minimization of $F_{t}$ for fixed moment of time $t$ :

$\forall u \in U\left[\forall 1 \leq i \leq N \quad F_{i}(t, u) \leq F_{i}\left(t, u_{0}\right)\right] \Rightarrow u=u_{0}$.

Here, we will consider the following three most fundamental criteria for each single decision step :

- the total yields on the interval $[t-1, t), F_{1}(t, u)$.

- the rate of risk, $\left(F_{2}(t, u)\right.$,

and

- the amount of liquidity available immediately at time $t, F_{3}(t, u)$.

To find an optimal investment strategy for the period of time $[0, T]$ we will request the decision-maker to formulate the additional information about his preferences in form of reference trajectories. Then we will interactively generate the nondominated solutions 
to (13) which fulfill the decision-maker's demands. The compromise strategy thus being found may be regarded as a result of the maximization of an a priori unknown utility function over the set of nondominated points.

A reference point is an element of the criteria space which represents the values of criteria being of a special importance to the decision-maker. A reference trajectory consists of reference points defined over certain planning period. In the above decision model the reference points may be results of investor's presumptions regarding the investment process, e.g. one can assume that $8 \%$ rate of return at a $3 \%$ risk rate with the confidence level $90 \%$ is a satisfactory solution, while the parameters $(12 \%, 1 \%, 99 \%)$ would be a desired solution. The methods of simultaneously applying multiple reference points to select a compromise decision in a multicriteria decision making problem has been studied in the recent years by the author Górecki, Skulimowski (1986), Górecki, Skulimowski (1988), Skulimowski, Schmid (1992).

Besides of the well-known desired values of criteria in the multiple reference points method one can consider also anti-ideal reference points, which achievement may be regarded as the failure, the solutions available at the pre-decision stage (or status-quo reference points), and the lower limits of optimality which determine the limits of the optimal values (if any). The reference points come as results of experts judgments independently from the statement of the multicriteria problem (13). The resulting model of decision-maker preferences (see Górecki, Skulimowski (1986) for more details) consists of two subsets of the criteria space $R_{-1}$ and $R_{1}$, aggregated as convex hulls of the neighboring classes of reference points, and two functions $g_{1}$ and $g_{-1}$ modelling the decision-maker's wish to reach the set $R_{1}$ and to avoid $R_{-1}$.

To select a solution, taking into account all above preference structure, we find the set D which contains all solutions nondominated in the sense of the maximization of $g_{-1}$ and minimization of $g_{1}$. Applying this method we reduce the decision problem to the bicriteria trade-off between the measures of proximity to the sets $R_{1}$ and $R_{-1}$. Thus we confine our interactive search for a compromise solution to the intersection of $D$ and of the set of nondominated points to the multicriteria optimization problem (13). This procedure will be repeated on-line for partial optimization problems ending at each moment $t, t \in$ $\left[t_{1}, T\right], t_{1}>0$.

\subsection{The trajectory optimization problem}

A general formulation of the multicriteria optimal control problem for the dynamical system described by difference equations may be presented as follows:

$x(t+1)=f(x(t), u(t)), x_{0} \in X_{0}, u(t) \in U(t)$ for $t_{0} \leq t \leq T$,

where $x(t) \in \mathbb{I R}^{n}, u(t) \in I R^{k}$, and the constraint set $U(t)$ is a closed and convex subset of $\mathbb{R}^{k}$. The classical multicriteria optimization task for the above system consists in simultaneous minimization of the functions $F(u):=\left(F_{1}(u), \ldots, F_{N}(u)\right)$ for the terminal time $T$. This formulation leads usually to finding a Pareto-optimal control $u_{o p t}$ satisfying some additional conditions resulting from the consideration of the decision-maker's preferences which are not included in the preliminary problem formulation.

In contradistinction to (13)-(14), a trajectory optimization problem (see Wierzbicki (1988)) involves the consideration of the values of $F(x(t), u(t))$ for the intermediary values of $t$, in the sense that they should be optimal for each moment of time. Thus this problem is transformed in fact into the multicriteria problem with an number of objectives 
equal to the number of time moments considered. The additional preference information may also be given in form of conditions concerning a subinterval of $\left[t_{0}, T\right]$, as e.g.

$F(u(t)) \in Q(t)$ for $t \in\left[t_{1}, T\right]$

where $Q$ is so-called reference multifunction for (13) - (15) and $t_{1} \in\left[t_{0}, T\right]$. An approach to solve a trajectory optimization problem, which has been admitted for portfolio optimization, consists in finding a finite sequence of time instances $t_{i} \in[0, T], i=1, \ldots k$, such that the original problem is equivalent to the multicriteria optimization problem with the objectives being the momentary values of $F(x(t), u(t))$ for $t:=t_{i}, i=1, . . k$. Further, in the reference output trajectory problem considered here to model the consumption demands, we have explored the dependence of the values of the remaining criteria on the consumption curve to reduce the dimensionality of the problem.

\subsection{Multiple reference point and reference trajectory method applied for dynamic portfolio optimization problems}

The solution of a portfolio optimization problem based on the above presented method can be undertaken in the following steps.

\section{The modelling stage.}

M1. The portfolio structure must be described symbolically using the prescribed format (cf. Skulimowski, Schmid (1992)). The system will automatically derive the state vector. Each state variable will have a fixed meaning available to the user as the interpretation vector.

M2. The transaction tables have to entered into the system. The transaction matrix will be created automatically.

M3. The price information must be supplied to the system.

M4. The planning period, the past data, and transaction constraints are to be specified by the user.

II. The goal setting and interactive solution procedure.

G1. The level of risk it a, desired revenues, and the amount of liquidity are to be predefined for the next series of interactive steps.

G2. The user defines its desired consumption curve as a reference trajectory over the planning period

G3. Step G2 is repeated for different consumption curves so as the revenues desired in step G1 were achieved at a risk level it a. If these goals cannot be achieved, the system automatically points out the time moments to update the consumption in the next interactive step.

G4. If no compromise solution can be found : step G1 is repeated for interactively modified values of risk, revenues, and liquidity levels.

III. The implementation of the decision suport system.

S1. Once a compromise decision is made, a corresponding sequence of optimal transactions is calculated by the system and communicated to the decision-maker. Missing states of environment are estimated using the generalized Kalman filtering techniques.

S2. The optimal values of all performance criteria are calculated and the results visualized on the screen. 


\section{DISCUSSION}

The portfolio modeling and optimization methodology here proposed may be regarded as a practical supplement of theoretical results on, compare e.g. the results presented in Östermark (1991), Perold, Sharpe (1988) and in the references cited therein. The presented portfolio optimization model would be especially well suited for short- and middle-range optimal strategy planning. It is expected that the best results can be achieved for calculating single-session strategies in case of securities trading. An implementation of long-term economical forecasts into the model, as e.g. the estimations of long-term price trends would provide a base for strategic decision support but would far exceed the framework of this study. The underlying idea while deriving this model has been a possibility of implementing it as a integrated interactive portfolio management and optimization package, in several versions fitting the needs of small through large investors. Some of the main elements of such package have been already implemented in the prototype versions:

- the description and coding of the portfolio structure in the state vector can be done with the tools provided by the system for the management and evaluation of quantitative information developed at the Institute of Computer Science, University of St. Gallen;

- the prototype of the decision support system based on multiple reference points, making also possible an application of reference trajectories and trajectory objectives, has been implemented including additional features especially suited for the portfolio management purposes;

- the databases, user interfaces, and the interface of the multicriteria decision support system to the implementation of the discrete-time stochastic control system has been established using standard software tools.

The database storing the sell-buy transactions, and determining the transaction plausibility by finding the demanded value of the margin requirement, which is especially important for options trading, has been coded as a part of a package developed by a commercial software company. The software package here presented can also be regarded as a supplement to other financial analysis products offered on the market.

\section{REFERENCES}

Górecki, H., Skulimowski, A.M.J. (1986) A joint consideration of multiple reference points in multicriteria decision-making. Found. Contr. Engrg., 11, No.2.

Górecki, H., Skulimowski, A.M.J. (1988) Safety Principle in Multiobjective Decision Support in the Decision Space Defined by Availability of Resources. Arch. Automatyki $i$ Telemech., 17, 68-87.

Östermark, R. (1991) Vector forecasting and dynamic portfolio selection : Empirical efficiency of recursive multiperiod strategies. it EJOR, bf 55, 46-56.

Perold, A.F., Sharpe, W.F. (1988) Dynamic Strategies for Asset Allocation. it Financial Analysts Journal, 16-27.

Skulimowski, A.M.J., Schmid, B.F. (1992) Redundance-free Description of Partitioned Complex Systems. Mathl Comput. Modelling, 16, 71-92.

Wierzbicki, A.P. (1988) Dynamic Aspects of Multi-Objective Optimization, in Multiobjective Problems of Mathematical Programming, held in Yalta, Oct. 26 - Nov. 2, 1988, Lecture Notes in Economics and Math. Systems, 351, Springer-Verlag. 